Original Article

\title{
Effects of conventional neurological treatment and a virtual reality training program on eye-hand coordination in children with cerebral palsy
}

\author{
Ji-won Shin, MS, PT ${ }^{1)}$, Gui-Bin Song, MS, PT ${ }^{2)}$, GaK Hwangbo, PhD, $\mathrm{PT}^{1)^{*}}$ \\ 1) Department of Physical Therapy, College of Rehabilitation Science, Daegu University: 15 Jillyang, \\ Gyeongsan-si, Kyeongbuk 712-714, Republic of Korea \\ 2) Department of Physical Therapy, Yeungnam University College, Republic of Korea
}

\begin{abstract}
Purpose] The purpose of the study was to evaluate the effects of conventional neurological treatment and a virtual reality training program on eye-hand coordination in children with cerebral palsy. [Subjects] Sixteen children ( 9 males, 7 females) with spastic diplegic cerebral palsy were recruited and randomly assigned to the conventional neurological physical therapy group (CG) and virtual reality training group (VRG). [Methods] Eight children in the control group performed 45 minutes of therapeutic exercise twice a week for eight weeks. In the experimental group, the other eight children performed 30 minutes of therapeutic exercise and 15 minutes of a training program using virtual reality twice a week during the experimental period. [Results] After eight weeks of the training program, there were significant differences in eye-hand coordination and visual motor speed in the comparison of the virtual reality training group with the conventional neurological physical therapy group. [Conclusion] We conclude that a well-designed training program using virtual reality can improve eye-hand coordination in children with cerebral palsy. Key words: Cerebral palsy, Virtual reality, Eye hand coordination
\end{abstract}

(This article was submitted Feb. 6, 2015, and was accepted Apr. 3, 2015)

\section{INTRODUCTION}

Cerebral palsy is a group of disorders that hinder the development of movement and posture, causing activity limitation. It can be attributed to nonprogressive disturbances occurring in the fetal or infant brain and is frequently characterized by difficulties with feeling, perception, communication, recognition, and behavior, as well as convulsions ${ }^{1}$. During normal development, babies learn the characteristics of objects through the sensations acquired by the hands until they develop their sight. They learn the size and characteristics of forms as their vision improves and develop their body schemes fully through all sensory channels via the learning relationship between bodies and the environment ${ }^{2}$. Limitation in the functional use of hands is the primary characteristic of the motor disturbance found among children with cerebral palsy, which is manifested in various forms according to the degree of damage from hindered brain development ${ }^{1}$. Eye-hand coordination movement is the skill whereby visual information from the eyes and motor information from the hands are efficiently applied

*Corresponding author. Gak Hwangbo (E-mail: hbgak@ daegu.ac.kr)

(C2015 The Society of Physical Therapy Science. Published by IPEC Inc. This is an open-access article distributed under the terms of the Creative Commons Attribution Non-Commercial No Derivatives (by-ncnd) License $<$ http://creativecommons.org/licenses/by-nc-nd/3.0/> . to work together as an essential component of self-help activities, play, perception, and school work ${ }^{3}$. In this regard, postural control is extremely important for the development of hand functions, while brain control affects the capacity of children to perform a visual search ${ }^{4}$. Therefore, problems with eye-hand coordination make it hard to accept various types of sensory information from the external environment and make it difficult for children to experience search tasks in their daily lives, such as playing and school activities ${ }^{5}$.

Virtual reality, an interactive simulation allowing users to have experiences similar to real-life ones with the help of computer hardware and software ${ }^{6}$ ) is a method for solving problems through the display of a series of interacting tasks on the screen in which the user appears through a video capture system ${ }^{7}$. Children taking part in virtual reality exercises are provided with visual and auditory feedback generated while they are performing specific tasks expressed in the form of games; the feedback enables them to start controlling their movements efficiently ${ }^{8)}$.

Among other rehabilitation research studies based on virtual reality, a study by Seo et al. ${ }^{9}$ ) in 2011 suggested that virtual reality is effective for improving visual sensory functions, in addition to the functional and muscular capacity of the upper limbs, in the case of stroke patients. Further studies by Levin et al. ${ }^{10)}$ and by Merians et al. ${ }^{11)}$ also found that virtual reality is effective for improving hand and upper limb functions in stroke patients. However, it was difficult to assess the continued effects of such rehabilitation activities, as it was not possible to observe whether the movements 
Table 1. General characteristics (mean \pm SD)

\begin{tabular}{lcc}
\hline & CG & VRG \\
\hline Gender (male/female) & $3 / 5$ & $6 / 3$ \\
Type of cerebral palsy (diplegia) & 8 & 8 \\
Height (cm) & $127.1 \pm 11.3$ & $128.1 \pm 5.8$ \\
Weight (kg) & $29.5 \pm 7.7$ & $30.0 \pm 6.3$ \\
Age (months) & $100.8 \pm 16.1$ & $106.8 \pm 2.5$ \\
Gestational age (weeks) & $30.8 \pm 1.6$ & $30.0 \pm 2.2$ \\
\hline
\end{tabular}

CG: conventional neurological physical therapy group; VRG: virtual reality training group

regulated at hospitals were properly performed at home. In addition, treatment outcomes were not good when patients were not interested in the treatments or had low expectations of them ${ }^{12}$. Mechling ${ }^{13)}$ reported that children with intellectual disabilities are able to employ their visual, auditory, and proprioceptive senses when playing with toys that generate sound and light, or bell sounds and vibrations; this also improves their motor outputs and focus. Herris and Reid $^{14)}$ suggested that virtual reality play is aimed at introducing motivation and has potential as a mediational means to measure the interest of research participants, particularly children with cerebral palsy. A study on the eye-hand coordination of children with cerebral palsy by Saavedra et al. ${ }^{15)}$ proposed that accurate arm stretching reflects the coordinated movements of the eyes, brain, and hands. Straker et al. ${ }^{16)}$ mentioned the need to provide dynamic video games for children with poor motor coordination who require motor development and physical activities. Accordingly, there is a need to investigate motor development and eye-hand coordination in children with cerebral palsy by providing treatment rooms and by using virtual reality programs as a mediatory means to induce interest and focus in them.

This study aimed to determine whether training with a Nintendo Wii virtual reality program can be used as a mediatory means that can affect eye-hand coordination by inducing interest in children.

\section{SUBJECTS AND METHODS}

The researchers chose 16 children with cerebral palsy from a group of patients visiting K Children's Development Center in Daegu, Republic of Korea, who had been diagnosed with palsy of both lower limbs, but did not have hearing and visual disabilities: they were able to communicate sufficiently to understand cooperate with the instructions of the researchers and were classified into stages 1 to 3 the Gross Motor Function Classification System (GMFCS). Of the 16 children, eight children whose exercise treatment only consisted of grasping objects by stretching their arms, in addition to other basic exercises, were selected as a conventional neurological physical therapy group (CG). Eight other children participated in the virtual reality program training and basic exercises, and they formed the virtual reality training group (VRG).

The research duration was eight weeks from July 26, 2014, to September 18, 2014, and it included twice-weekly
Table 2. Comparison of eye-hand coordination between before and after the intervention within each group shown as the mean $\pm \mathrm{SD}$ (unit: score)

\begin{tabular}{lcccc}
\hline & \multicolumn{2}{c}{ CG } & \multicolumn{2}{c}{ VRG } \\
\hline & Pretest & Posttest & Pretest & Posttest \\
\hline EHC & $150.0 \pm 15.4$ & $167.8 \pm 12.9^{*}$ & $129.0 \pm 47.0^{*}$ & $143.5 \pm 36.4^{*}$ \\
VMS & $17.5 \pm 8.2^{*}$ & $26.9 \pm 7.5^{*}$ & $15.5 \pm 9.7^{*}$ & $22.8 \pm 10.4^{*}$ \\
\hline
\end{tabular}

*Significant difference from the pretest at $<0.05$. ${ }^{\text {a }}$ Significant difference in gains between two groups at $<0.05$. CG: conventional neurological physical therapy group; VRG: virtual reality training group; EHC: eye-hand coordination; VMS: visual-motor speed

sessions of 45 minutes per session.

The eye-hand coordination and visual-motor speed, which improve motor intervention, subtests of the KDTVP-2 (Korean-Developmental Test of Visual Perception) were conducted as a preliminary evaluation to measure eyehand coordination. A post-test evaluation was conducted after tests were carried out for eight weeks on both groups. The tests included 45 minutes of exercise treatment for the CG group, and 45 minutes of exercise treatment along with 45 minutes of Nintendo Wii game play for the VRG group.

For the CG patients, the basic exercises, including articular movement exercises, muscular extension and strength enhancement exercises, and trunk stabilization exercises, took 30 minutes. The other exercises included grasping objects by stretching the arms in various directions. The aim of this exercise was to grasp objects held by the therapist by stretching the arms while sitting with the trunk perpendicular to the floor. The idea is to maintain the body's centerlines, grasp objects by stretching the arms while breaking away from the centerline, return back to the original posture, all within 15 minutes. Like the CG patients, the VRG patients performed 30 minutes of basic exercises while taking practice based on upper limb movements. This basic exercise involved movement based on programs, for example, hitting targets using one or both hands while sitting in a posture, taking account of the engagement and movement of children, moving in directions according to instructions, discerning pictures, and so on.

SPSS Ver. 12.0 for Microsoft Windows was used for statistical processing of the data.

The Mann-Whitney U test was used to establish the significance between the CG and VRG groups, and Wilcoxon's signed-rank test was used for in-group comparisons between pretreatment and post treatment values among the participants.

The statistical level of significance was $\alpha<0.05$.

\section{RESULTS}

Neither the CG nor the VRG groups showed general Characters (Table 1).

Both the CG and VRG groups saw significant changes in eye-hand coordination and visual motor speed after the interventions $(\mathrm{p}<0.05)$ (Table 2).

A comparison of the two groups before and after the inter- 
ventions showed no significant differences between the two groups for eye-hand coordination and visual motor speed ( $p$ $>0.05$ ) (Table 2).

\section{DISCUSSION}

This study aimed to look at the impact of training on eyehand coordination in children with cerebral palsy by inducing interest in the children using a virtual reality program. The study selected children between 4 and 8 years old, with the control group participating in grasping exercises in various directions and the test group participating in Nintendo Wii-based virtual reality programs, in addition to basic exercises, for a period of eight weeks. The effects were verified by carrying out an evaluation using the K-DTVP-2 before and after the interventions.

Comparisons between evaluations before and after the interventions revealed statistically significant results in terms of changes in eye-hand coordination, visual-motor speeds, and visual-motor speed age in both the CG and VRG groups in the study. These results correspond to results from existing studies, including one regarding the impact of visual-motor development programs on the visual perception capabilities of infants, which showed an improvement in their visualmotor coordination capabilities. A further study looked at the impact of visual perception training programs in terms of how they affect improvements to visual perception capabilities (eye-hand coordination and visual-motor speeds ${ }^{17}$ ). The study of Hong ${ }^{18)}$ on integrated sensory treatment showed improved results in terms of visual-motor integrated capabilities including eye-hand coordination and visual-motor speeds.

There was no significant difference in eye-hand coordination and visual-motor speeds between the CG and VRG groups in this study. This result differs from that of a study by Chung ${ }^{17)}$ on the impact of visual-motor development programs on the visual perception capabilities of infants; that study showed significant differences between groups, as the study included visual perception training programs for its VRG, while it was not included for its CG. By comparison, the current results indicate that the CG group, which performed grasping exercises by stretching their arms, and the VRG group, additionally performed the VR programs, both displayed within-group differences between the results before and after the tests but no significant difference between the groups; in other words, both activities had a positive impact on improvement of eye-hand coordination. Furthermore, a comparison between the results before and after the interventions in each group showed significant differences in the eye-hand coordination scores for the CG group. It is thought that the significant results in terms of eye-hand coordination scores indicate a positive impact on eye-hand coordination with age. Visual perception capabilities are the most important and fundamental perception capabilities throughout early childhood and can affect self-help activities, work, play, and leisure activities in infants ${ }^{19}$. Handwriting clarity in children between five and six is reported to be correlated with eye-hand coordination in visual-motor coordination activities requiring mobility, such as drawing by tracing, and with visual-motor speeds. Visual perception development generally follows as children grow up, with most children continuing to develop skills up to the age of 9 and completing their development by the age of 10 or $11^{20)}$. Although the average age of the participants in this study was 100.8 months (8 years and 3 months) for the CG and 106.8 months ( 8 years and 8 months) for the VRG, a comparison of speed age between before and after tests of visual-motor capabilities showed changes from 63.25 months (5 years and 2 months) to 80.5 months ( 6 years and 6 months) in the CG, and from 65.25 months (5 years and 4 months) to 88.5 months (7 years and 3 months) in the VRG. In these cases, it was found that the visual-motor speed age was lower than the real ages of the children and that there were increases in visual-motor speed ages after mediation; it follows that there were also significant mediations in both groups. Therefore, children's visual perception development is of the utmost significance in enabling them to live as humans, and visual perception development may be even more crucial to children with cerebral palsy who have disabilities in terms of their eye-hand coordination function, along with motor disabilities ${ }^{17}$.

Among previous research studies on the use of VR programs for children with cerebral palsy, a study by Deutsch et al. ${ }^{21)}$ reported on the feasibility of VR for treating children with palsy of both lower limbs and found that there were improvements in visual perception processing, posture control, and functional movement capabilities. Another study by Yoo et al. ${ }^{22}$ found that there were changes in neural plasticity related to self-help activities after the VR program was applied as part of neural rehabilitation mediation therapy for strengthening exercise performance in children.

Many changes occur when children with cerebral palsy reach school age after early childhood. For example, they are exposed to fewer hours of treatment due to a combination of schools, private institutions, and treatment rooms following their intensive early childhood treatment period, which is centered on daytime wards at hospitals. Furthermore, they are faced with many self-help activities in their daily lives and exposed to self-study environments following intensive play and care at home, in addition to nursery facilities. This period requires a positive influence on functional improvement of children through the integration of home life with therapy activities in treatment rooms. The current study proposes training methods that are VR based and provide using the Nintendo Wii, as such devices are easy to use in terms of cost and are highly efficient as methods for enhancing treatment effects. Furthermore, we believe that VR training and exercises such as grasping objects by stretching the arms can be employed as useful activities for school age children, as they positively affect eye-hand coordination and visual perception capabilities, which are relevant to the improvement of handwriting skills and cognitive learning in children. Also, the program used in this study offers the potential to be used as a home program connected to treatment rooms by inducing interest in school age children with the appropriate assistance of VR programs.

However, there were limitations in this research in that the number of participants was small and control factors other than the test conditions in the daily lives of individual children could not be unified. Moreover, it is difficult to 
generalize the results from tests based on children with spastic palsy of both lower limbs to all children with cerebral palsy. Direct research should be conducted in the future to determine home virtual reality programs based on the results of this study.

\section{REFERENCES}

1) Bax M, Goldstein M, Rosenbaum P, et al. Executive Committee for the Definition of Cerebral Palsy: Proposed definition and classification of cerebral palsy, April 2005. Dev Med Child Neurol, 2005, 47: 571-576. [Medline] [CrossRef]

2) Yalcinkaya EY, Caglar NS, Tugcu B, et al.: Rehabilitation outcomes of children with cerebral palsy. J Phys Ther Sci, 2014, 26: 285-289. [Medline] [CrossRef]

3) Jeong HW: The Effects of Visual Perception Training Program on the Visual Perception and Eye-hand Coordination Skills of Children with Spastic Cerebral Palsy, Dankook university Graduate school of special education. 2008

4) Bertenthal B, Von Hofsten C: Eye, head and trunk control: the foundation for manual development. Neurosci Biobehav Rev, 1998, 22: 515-520. [Medline] [CrossRef]

5) Case-Smith J: Occupational Therapy for Children, 4th ed. Mosby, 2001.

6) Cho GH, Hwangbo G, Shin HS: The effect of vitual reality-based balance training on balance of the elderly. J Phys Ther Sci, 2014, 26: 615-617. [Medline] [CrossRef]

7) Weiss PL, Rand D, Katz N, et al.: Video capture virtual reality as a flexible and effective rehabilitation tool. J Neuroeng Rehabil, 2004, 1: 12. [Medline] [CrossRef]

8) Flynn S, Palma P, Bender A: Feasibility of using playstation 2 gaming platform for an indivisual poststroke: a case report. J neuronal. Phys Ther, 2007, 31: 180-189.

9) Seo SM, Song CH, Lee K, et al.: Video Game-Based Exercise for upperextremity function, strentgth, visual perception of stroke patients. J Spec Educ Rehabil, 2011, 55: 155-180.

10) Levin MF, Knaut LA, Magdalon EC, et al.: Virtual reality environments to enhance upper limb functional recovery in patients with hemiparesis. Studies in health technology and informatics, 2009, 145: 94-108.

11) Merians AS, Tunik E Adamovich SV, et al.: Virtual reality to maximize function for hand and arm rehabilitation: exploration of neural mechanisms. Studies in health technology and informatics, 2009, 145: 109-125.

12) Burdea GC: Virtual rehabilitation - benefits and challenges. Methods Inf Med, 2003, 42: 519-523. [Medline]

13) Mechling LC: Comparison of the effects of three approaches on the frequency of stimulus activations, via a single switch, by students with profound intellectual disabilities. J Spec Educ, 2006, 40: 94-102. [CrossRef]

14) Harris K, Reid D: The influence of virtual reality play on children's motivation. Can J Occup Ther, 2005, 72: 21-29. [Medline] [CrossRef]

15) Saavedra S, Joshi A, Woollacott M, et al.: Eye hand coordination in children with cerebral palsy. Exp Brain Res, 2009, 192: 155-165. [Medline] [CrossRef]

16) Straker LM, Campbell AC, Jensen LM, et al.: Rationale, design and methods for a randomised and controlled trial of the impact of virtual reality games on motor competence, physical activity, and mental health in children with developmental coordination disorder. BMC Public Health, 2011, 11: 654 [CrossRef]. [Medline]

17) Chung HS: The Effect of Visual-Motor Development Program on Developing Visual Perception of Preschool Children, Department of Physical Education of The Graduate School Ewha Womans University, 2003.

18) Hong EK: The effects of sensory integration intervention for postural control and visual-motor integration. J Spec Educ Rehabil Sci, 2009, 48: 77-99.

19) Ochi A, Morioka S, Kataoka Y, et al.: stroke patient's perception of an inclined sitting support surface: a comparison between visual and proprioceptive input. J Phys Ther Sci, 2008, 20: 45-49. [CrossRef]

20) Cho K, Yu J, Jung J: The effect of virtual reality based rehabilitation on upper extremity function and visual perception changes in stroke patients. J Phys Ther Sci, 2012, 24: 1205-1208. [CrossRef]

21) Deutsch JE, Borbely M, Filler J, et al.: Use of a low-cost, commercially available gaming console (Wii) for rehabilitation of an adolescent with cerebral palsy. Phys Ther, 2008, 88: 1196-1207. [Medline] [CrossRef]

22) You SH, Jang SH, Kim YH, et al.: Cortical reorganization induced by virtual reality therapy in a child with hemiparetic cerebral palsy. Dev Med Child Neurol, 2005, 47: 628-635. [Medline] [CrossRef] 